\title{
Regulatory Disincentives for Developing Antibiotics for Common Indications?
}

\author{
Guy W. Amsden, PharmD
}

\author{
Corresponding author \\ Guy W. Amsden, PharmD \\ Department of Pharmaceutical Care Services, Bassett Healthcare, \\ One Atwell Road, Cooperstown, NY 13326, USA. \\ E-mail: guy.amsden@bassett.org
}

Current Infectious Disease Reports 2007, 9:29-34

Current Medicine Group LLC ISSN 1523-3847

Copyright (C 2007 by Current Medicine Group LLC

Pediatric and adult community-acquired respiratory tract infections remain some of the most common reasons for visits to primary care practitioners, and the antibiotics used to treat them are historically highly profitable for their manufacturers. Despite these facts and the continued evolving need for new treatments for these infections, virtually no new agents have been developed in the past decade. This review explores some regulatory guidelines that could potentially explain the dearth of development, and it provides some practical answers for resolving them.

\section{Introduction}

Adult and pediatric community-acquired respiratory tract infections (CARTIs) include a variety of infections commonly encountered by clinicians on a daily basis and on a global scale including acute otitis media (AOM), acute bacterial sinusitis (ABS), pharyngitis/tonsillitis, bronchitis, acute exacerbation of chronic bronchitis (AECB), and community-acquired pneumonia (CAP). The responsible pathogens for these infections include a plethora of viruses, typical bacteria, and atypical bacteria such as Streptococcus pneumoniae, Streptococcus pyogenes, Haemophilus influenzae, Moraxella catarrhalis, Mycoplasma pneumoniae, Chlamydophila pneumoniae, and less commonly Legionella pneumophila and Staphylococcus aureus. Regardless of appropriateness, especially in the United States, it is likely that patients presenting with these CARTIs will receive prescriptions for antibiotics that have recognized coverage of the most likely bacterial pathogens. As a result, historically, antibiotics marketed for these indications have been highly profitable for their manufacturers, with some marketed in the last decade producing well in excess of $\$ 1$ billion in annual sales. This profit has been especially true if a manufacturer markets both oral and intravenous formulations to allow for step-down therapy, and/or if they achieve regulatory approval for both adult and pediatric populations.

Patent expiration and generic competition was historically a death-knell for a manufacturer. However, this traumatic financial loss is blunted in some cases as brand companies purchase/start generic houses to compete for the generic market of their previously branded product. In sharp contrast to the financial bonanza associated with CARTI antibiotics, those developed and marketed for nosocomial pathogens/infections have been associated with revenue generation approximately a log-fold lower, though this difference has moderated a bit in recent years. As a result, although a popular CARTI antibiotic can produce an annual revenue stream well in excess of its development costs, even popular nosocomial antibiotics may take multiple years to recapture development costs, let alone show a profit.

Therefore, in the past, pharmaceutical companies that have anti-infective divisions have heavily favored development of antibiotics targeted at CARTIs, with the number of new antibiotics for CARTIs significantly outnumbering those introduced for nosocomial indications. Over the last 10 years, however, this trend has radically reversed, as evidenced by only one antibiotic (ie, telithromycin) being approved and widely marketed for CARTIs, as opposed to several agents (eg, ertapenem, linezolid, daptomycin, dalbavancin, tigecycline) being approved or about to be approved for nosocomial/ resistant pathogen indications. Although such a reversal may be understandable if it was only because the previously typical variety of new CARTI agents were still in development, this is not true, as has been lamented internationally. Rather, almost all anti-infective development has shifted away from CARTI agents to nosocomial/ resistant pathogens, fungi/yeast, and most especially viruses, even though these markets pale in comparison to the potential revenue stream of launching a new CARTI agent. Even though these additional areas undoubtedly need more research and drug development, the utter lack of development of new CARTI agents defies logic when one considers at the very least the potential financial benefits for these for-profit companies. 
This article explores some potential answers as to why this trend is happening with CARTI agents and proposes some novel, albeit potentially unpopular, remedies to break the impasse to developing these needed drugs.

\section{Do We Really Need More CARTI Antibiotics?} There are at least two sides to every discussion, and this one is no different. Some may argue that vaccine development for prevention of infection with the most common pathogens and their most common strains will significantly decrease the need for treatment of CARTIs (eg, otitis media, epiglottitis, CAP) and as a result the need for new CARTI antibiotics. Proponents of this line of thinking would point to the successes noted to date with the meningococcal, pertussis, $H$. influenzae type $\mathrm{B}$, and polysaccharide and conjugate pneumococcal vaccines.

Undeniably, the introduction of these vaccines has decidedly curbed invasive disease and the resultant morbidity and mortality associated with these pathogens, but their benefits should not be overestimated. Using the heptavalent pneumococcal conjugate vaccine as an example, initial large-scale surveillance through the U.S. Centers for Disease Control and Prevention's (CDC) Active Bacterial Core Surveillance system demonstrated a $29 \%$ reduction in invasive pneumococcal disease overall as opposed to before the vaccine was marketed [1]. The differences were most marked in those people younger than 2 years old, who demonstrated a $69 \%$ decrease. The protection seemed to be transmitted via a herd effect through adult age groups to lesser extents $(32 \%$ in 20 - to 39 -year-olds and $18 \%$ in those older than 65 years of age). Not only did this study demonstrate a $78 \%$ decrease in disease caused by vaccine serotypes and $50 \%$ by related serotypes, but other studies published around the same time and since have also demonstrated decreased carriage and transmission of resistant pneumococcal strains [2-4].

Although these data seem to indicate that pneumococcal infections should rapidly become an insignificant concern, many of these same studies pointed out that the incidence of colonization and invasive infection by once uncommon nonserotype strains are significantly increasing $[4,5]$. As such, even though science may be moving in the right direction to suppress the strains that have been problematic for so long, this same science is unleashing strains that could be as troublesome as the ones that have been suppressed. Investigators are waiting to see just how much danger these strains will pose. Obviously, since it takes upwards of 7 or more years to bring a new compound to market, researchers cannot wait to develop new antibiotics or classes of antibiotics until this paradox is solved. To do so could be disastrous on a global level. Disaster may be even closer at hand if one takes into account the troubling documentation of communityacquired methicillin-resistant $S$. aureus in CARTIs and the demonstration of rapid resistance development and subsequent clinical failure in pneumococcal infections treated with fluoroquinolones [6-8].

\section{What Causes this Dearth of Development?}

The answer to this question is also multifactorial. Undoubtedly, some companies have shifted their internal focus away from anti-infectives to therapeutic areas in which they may have greater strength, such as oncology or cardiology. But this shift fails to explain the trend for companies that have had strong historical presence in the CARTI antibiotic market. Because the demand for these types of antibiotics has not dramatically dropped in the last 10 to 20 years, and because multiple companies exhibit the same decreased development trend, one could infer that some seminal regulatory shift has occurred.

In the past, creating new criteria for the development of a new antibiotic has made considerable sense. The best example of this probably has been the clarification of lower respiratory tract infections (LRTIs). Prior to 1992, an antibiotic could have been studied in any pneumonia population or even a mixed population and be approved with a general pneumonia or LRTI indication. As the variety of LRTI syndromes became well recognized, it became wholly appropriate to break out the various LRTI types and require investigation in those populations before an antibiotic could receive approval for that indication. Since this distinction became the norm, antibiotics have been labeled for CAP or nosocomial pneumonia as appropriate.

This recognition and subsequent separation of syndromes within syndromes continues today at the advisory level of the U.S. Food and Drug Administration (FDA). For example, recent discussions have addressed whether patients with multiple occurrences of an infection like otitis media should be considered separately from children that may have only 1 or 2 cases of AOM per year [9]. The reasoning behind this suggestion is that the pathology of the infection and penetration of an antibiotic could change with frequent, repeated infections of the middle ear space (eg, increased scarring of the area, decreased penetration). The same argument could be set forth for other CARTIs including sinusitis where there is an obvious difference in overall treatment and outcomes between patients who have an occasional bout of sinusitis and those that have sinusitis multiple times per year. These patients often require surgical intervention and antimicrobial therapy.

The separation of distinct syndromes within an indication certainly makes good medical and scientific sense and assures that patients with the respective syndromes receive the most appropriate and targeted antibiotic, and these issues do not seem to be causing the angst and disconnect in the drug development community. Rather, a significant contribution to the decline in CARTI antibiotic development appears to be the focus of these guideline development advisory groups on scientific purism and idealism for future CARTI antibiotic approvals as opposed to medical, 
scientific, and arguably ethical realities. As an example, discussions on clinical trial design for AOM and ABS have included serious suggestion of serial tympanocentesis and sinus puncture to identify the pathogen, monitor its speed of eradication, and document eradication [9,10]. Proponents of this design argue that it will allow for documenting what pathogens are being treated, assuring that a bacterial pathogen is involved, assuring that it is susceptible to the study drugs, and assuring that the pathogen is eradicated, regardless of clinical outcome $[9,10]$.

Although all these issues are valid and the proposed methods may be the most academically pure, one must ask whether the FDA should request them or mandate them. Also, what will these methods change in terms of the use of these antibiotics, and should the manufacturer be responsible for developing this knowledge base and dictate proper clinical practice? With so much criticism about conflict of interest throughout drug development and its regulatory oversight, perhaps a relatively unbiased group (eg, the U.S. National Institutes of Health or its foreign equivalents) would be a better sponsor than one with a very heavily vested interest (ie, the manufacturer).

However, the most important question is whether these in-depth questions and idealistic methods will change results. For example, along with AECB, CAP is a CARTI with significant associated morbidity and/or mortality if treated with inappropriate anti-infectives. Even though CAP mortality has been significantly lower since the introduction of systemic antibiotics in the late 1940s ( 30 deaths/100,000 cases vs more than 170 deaths $/ 100,000$ cases prior), mortality rates have not improved significantly since then, regardless of which new drugs and drug classes have been introduced [11].

Importantly, though, they also have not gotten worse since the age of antibiotic resistance intertwined with the age of antibiotics. This development is most likely due to the disconnect between resistance and clinical outcomes with the most common CARTI pathogen, the pneumococcus, and classes of antibiotics such as the $\beta$-lactams and macrolides. To this day, in parts of the world with the worst resistance problems, surveillance studies in hospitalized CAP patients show that if drugs are dosed appropriately, no significant mortality difference exists between patients treated with a drug or combination of drugs that have a susceptible isolate and those with a resistant one $[12,13 \bullet, 14]$. This result has been demonstrated not only in CAP patients but even in patients with meningeal disease, which is inherently harder to treat and from which it is harder to fully recover [15].

Trials studying new antibiotics for CAP require an attempt to identify the active pathogen, but through an indirect method (sputum expectoration). Not only does this method offer a poor return in terms of identifying a pathogen $(<50 \%)$, it is also fraught with two problems: significant contamination by epithelial cells making the samples ineligible for culture, and false positives when the nasopharynx is chronically colonized with common pathogens. Based on these limitations, it is generally recognized that sputum culturing for CAP is neither cost effective in clinical practice nor helpful in guiding appropriate therapy. The continued regulatory guidance suggesting its use for CAP trials despite the determination that it is suboptimal seems counterintuitive, especially when comparing it to ideal textbook methods being recommended for culturing of CARTIs, which are now rarely associated with mortality or significant complications.

To apply the same standards to CAP and AECB trials that are being proposed for AOM and ABS trials, one would mandate baseline and serial bronchoscopies and/or bronchoalveolar lavages in all studies. If one were to consider one expert's suggestion to decrease sinus punctures from two to one by inserting and maintaining a sinus catheter for follow-up culturing [10] and extrapolate that suggestion to CAP/AECB trials, a patient would actually have to be intubated during the initial bronchoscopy to allow transtracheal aspiration during follow-up rather than undergo another bronchoscopy.

Obviously conducting one-let alone serial-bronchoscopies for future AECB/CAP antibiotic development appears extreme; it is a direct analogy to the AOM/ABS guidelines being developed. Even though in the past, some of these procedures (ie, tympanocentesis) may have been done with some regularity by an average clinician, now pediatricians, family medicine practitioners, internists, hospitalists, or emergency department doctors rarely ever perform these procedures or have the necessary skill sets to do so. Because these types of practitioners, not specialists, have the average AOM, ABS, AECB, and CAP cases presented to them, the requirement of these procedures for drug development trials would necessitate that specialists (ie, otolaryngologists) or a very select group of investigators perform all trials. Limiting the trials to a select group of investigators inherently biases results as potentially not representative of the population as a whole or of the population in the country in which the study will be used for labeling.

Since the patients referred to subspecialists would be those with multiple occurrences of the infections within a short time, the results of studies done in these patients would not be representative of the AOM or ABS population as a whole. Rather, they would represent the chronic subpopulation under consideration as a separate indication-people who are either microbiologically or anatomically susceptible to this repetitive infection pattern.

Ideally, only patients with truly bacterial pathogens should be enrolled in trials and/or treated clinically, but the desire to assure this for regulatory approval appears more like a desire for appropriate antibiotic prescribing. This is more of a public health issue, and although it is highly desirable, it is not very reflective of real life. As it is not common medical practice to do tympanocentesis or sinus puncture, it is key to know what the potential 
pathogens are and how the antibiotic works against them. Though it will be argued that these invasive techniques can acquire that knowledge, one needs to ask whether it is even necessary any longer. With the exception of having a greater appreciation in recent years about the role of atypical pathogens in LRTIs, the responsible bacterial pathogens for CARTIs have not changed in recent history. By studying new drugs against those with proven efficacy in these indications, it can be directly extrapolated that any new treatment also has efficacy against them if its outcomes are equivalent or superior. If they are inferior despite in vitro preclinical testing, then more invasive procedures would be appropriate to determine the reason for its ineffectiveness (eg, penetration of an area), if continued studies were even considered ethical.

The issue of resistance and continued action of a drug can also be extrapolated as previously discussed (ie, if the antibiotics continue to work in pneumonia, they are likely to work for AOM or ABS if dosed correctly). From a regulatory standpoint, most trials have difficulty identifying more than a handful of resistant isolates against which efficacy can be judged. However, it is common sense and statistical probability that these antibiotics are being used in trials or in practice against resistant isolates on a daily basis, purely because the pathogens of most interest are the most common pathogens globally for all CARTIs. In addition, the resistances of concern approach $50 \%$ incidence. As such, if a drug has a problem with resistance, the problem would quickly become noticeable in everyday practice and would not require statistical manipulation or a case series to prove. No drug is $100 \%$ effective, even against a fully susceptible isolate, and so it makes one wonder whether isolates that have more resistance and are clinical failures are truly the result of poor drug activity or rather of the specific anatomy/pathology of a subset of patients who are more susceptible to retaining bacteria or developing biofilm-type patterns.

This variation on thinking may help explain why some experts have noted that the negative predictive value of the double tympanocentesis method may be as low as $32 \%$ [16]. It is also most likely a further example of the overestimation of the role of the antibiotic as the sole deciding factor for clinical success or failure in these cases, as opposed to the myriad aspects, both host and pharmacologic, of an infectious process. This issue is especially true when one considers the significant spontaneous resolution rates of many CARTIs, even those of bacterial origin $[9,10]$. It is tempting to analyze an antibiotic's comparative effectiveness only once a spontaneous resolution "correction factor" is taken into account, but a correction factor would have to be prospectively validated across a CARTI population as a whole and for the various regions within which the drug may be studied and marketed. Until this correction factor is fully prospectively validated, any analyses or reanalyses based on anecdotal corrections are of limited validity or interest.
A final issue associated with new guideline development is the ethics of mandating them. This issue of ethics is especially prominent when one considers the following factors: the low negative predictive value of some of these invasive procedures; the already established identification of the most likely viral or bacterial pathogens; the fact that the procedures are no longer part of standard care nor are they recommended outside the expertise of specialists; the inability of these procedures to alter therapy in a trial or practice, except maybe in chronic cases; and that the goal of the patient, parent, and clinician is clinical relief/resolution rather than identification of the exact pathogen [16-19].

Proponents of these invasive procedures find no harm in them and minimize concern over them, but their own descriptions betray the procedures' purported innocuousness, especially when one considers that patients typically must undergo conscious sedation and physical restraint to endure them $[9,10]$. Clinicians have sworn to first "do no harm," and there is a very low likelihood that the performance and/or repeat of any of these procedures will alter treatment or the clinical course in the average patient. Therefore, the idea of putting a patient, be it child or adult, through one of these painful invasive procedures, even with a modicum of reimbursement, approaches unethical behavior and is contrary to a clinician's oath.

Despite comments to the contrary, the only thing analogous between a repeat tympanocentesis or sinus puncture for documentation of eradication and a repeat urine culture to show urinary tract infection eradication is the demonstration of eradication. The similarity ends there, because one involves a painful invasive procedure on a healed or healing anatomic space under a modicum of conscious sedation and physical restraint, and the other involves a normal bodily function being performed into a specimen cup.

Although placebo-controlled trials for some CARTIs could be justified from a scientific standpoint, the studies in many countries (ie, the United States) would be exceedingly hard to enroll, especially if they involve children. This difficulty is due to the patient's or parent's perceived need for active intervention regardless of whether there is good evidence of the probability of a nonbacterial pathogen or of spontaneous resolution with symptomatic therapy.

\section{Are New Trial Guidelines Necessary?}

Despite the above commentary, new guidelines are most likely necessary and would be welcomed by the scientific and clinician communities. Although bringing in scientific experts to help formulate guidelines is a key and wellestablished practice, it is important to include experts with divergent scientific opinions, as opposed to complementary ones, so as to ensure all aspects of an issue are dealt with appropriately. Also, the experts must not benefit disproportionately from influencing the development 
of a guideline in one direction or another. For example, if Procedure $\mathrm{X}$ is only conducted at five sites on a regular basis in countries whose data would be acceptable for regulatory purposes, invited experts from those sites would disproportionately benefit from establishing Procedure X as a regulatory mandate.

Rather, similar to the FDA's 1992 invitation for comment from the Infectious Diseases Society of America, groups that span the whole issue should be invited, so research guidelines are relevant and reflective of average clinical practice, and so the data garnered from the study designs are acceptable to all potential users. For example, for otitis media, representatives from national and/or international societies for infectious disease, pediatric, family practice, and otolaryngology should be included at the very least.

Unlike the establishment of research practices contrary to current diagnostic and treatment guidelines, no clinician would argue that the wide overuse of antibiotics for bouts of CARTI that are likely viral in origin would benefit from moving criteria in trials toward more appropriate use. As such, as has been suggested, research trials should include symptomatic inclusion and exclusion criteria that would significantly increase the chances that a bacterial form of the CARTI in question is being treated $[9,10,19]$. Although these trials may require greater numbers of enrollees than a pathogen identification trial, they would reflect clinical diagnostic methods, support appropriate antibiotic use, and represent a solid test of a new antibiotic. These clinical comparator tests with tighter enrollment guidelines would hopefully strengthen noninferiority-type trials, especially if there is regulatory emphasis on a true appropriate comparator both in terms of dose and comparator choice. FDA could ensure this comparator appropriateness if the design of proposed regulatory trials is discussed prospectively, not only with the manufacturer proposing the study but also with the clinical associations impacted by the agent's introduction. Rather than a drug being approved on a study comparing Drug X to an agent approved for that indication or a historical dose of that agent, the regulatory approval studies could be timely comparisons that would be a truer test of the drug's efficacy and would provide clinically useful data immediately upon its introduction.

Although issues such as when to judge primary efficacy are still the subject of significant debate, only time will tell whether the suggestion of reversal from the typical end-ofstudy evaluations to end-of-therapy is appropriate or not. As it is uncommon for an average patient to have repeated infectious events within 2 to 4 weeks of a previous one, additional debate may be necessary to determine whether this reversed primary efficacy timepoint is truly appropriate for all patients in trials, or whether after action is taken to further separate out acute versus chronic versions of some of these CARTIs, it may only be appropriate for the latter definition.
The use of pharmacokinetic and pharmacodynamic (PK-PD) integration is and should be supported for the development of anti-infectives, but the breakpoints and other interpretation of these data appear to be oversimplified in some cases. In these studies, investigators must ensure that the animal models used as the basis are immunocompetent and that the sampled biomatrices in animals and humans are reflective of the pharmacokinetics of the drug in question. Too often, data developed use serum or plasma data, which may not be reflective of antibiotic activity at the infection site if it has significant distribution and/or uptake by phagocytes. In addition, site-specific pharmacokinetic testing should be performed in the treated population rather than healthy volunteers. This consideration can help ensure that the concentrations of drugs, especially any classes that may have their distribution affected by inflammation (eg, fluoroquinolones or macrolides), are accurate for the CARTI scenario justified by the PK-PD data [20].

\section{Conclusions}

There must be movement between industry, regulatory authorities, and experts, so that the development of agents to treat CARTIs is reinvigorated and reprioritized. Although some of the earlier suggestions for change or lack thereof may sound naive, it is important to remember that the CARTIs in question have been studied at great length for decades, and much is known about them already. As such, it is not in the interest of the clinical, scientific, and patient communities for regulatory authorities to continuously "reinvent the wheel" when it comes to straightforward indications such as these. Rather, with the exceptions of resistance and possible subsyndromes within the general CARTI classifications, not much has changed recently for any CARTIs except potentially the LRTIs.

Also, it seems counterintuitive to laud mandates for idealistic invasive culturing methods for CARTIs associated with minimal morbidity or mortality when regulatory guidelines allow far less sensitive or specific culturing options for CARTIs with significant morbidity and mortality risks (eg, CAP and AECB). The adoption of some of these changes such as subclassifying more recalcitrant versions of the CARTIs into separate indications, developing guidelines in line with clinical practice rather than scientific purism, and making clinical enrollment criteria stricter to assure that more bacterial rather than viral infections are being treated may help some of the issues that are stymieing development. Undoubtedly, though, this review has missed key issues, and the introduction of these ideas may introduce new issues to resolve. Monitoring resistance patterns and funding studies to do these invasive procedures to better understand the pathology of infections and their response to treatment are important, but they should be funded via a pathway that lends itself to less bias such as through NIH, CDC, or professional 
societies. Once findings from these studies are considered necessary for inclusion in discussions concerning treatment guidelines or regulatory development guidelines, they should be discussed in an open atmosphere with the involved clinical societies and regulatory authorities so that a cohesive conclusion may be developed and disseminated.

\section{Acknowledgment}

The author would like to acknowledge potential conflicts of interest. He has been a consultant for Pfizer Inc. (New York, NY, USA) and Pliva d.d. (Zagreb, Croatia), and he is doing or has done anti-infective research for Pfizer, Pliva, Bayer Corp. (West Haven CT, USA), Bristol-Myers Squibb (New York, NY, USA), and GlaxoSmithKline (London, UK).

\section{References and Recommended Reading}

Papers of particular interest, published recently, have been highlighted as:

- Of importance

- Of major importance

1. Whitney CG, Farley MM, Hadler J, et al.: Decline in invasive pneumococcal disease after the introduction of protein-polysaccharide conjugate vaccine. $\mathrm{N} \mathrm{Engl} \mathrm{J} \mathrm{Med}$ 2003, 348:1737-1746.

2. Dagan R, Givon-Lavi N, Zamir O, et al.: Effect of a nonavalent conjugate vaccine on carriage of antibioticresistant Streptococcus pneumoniae in day-care centers. Pediatr Infect Dis J 2003, 22:532-539.

3. Givon-Lavi N, Fraser D, Dagan R: Vaccination of daycare center attendees reduces carriage of Streptococcus pneumoniae among their younger siblings. Pediatr Infect Dis J 2003, 22:524-531.

4. Kyaw MH, Lynfield R, Schaffner W, et al.: Effect of introduction of the pneumococcal conjugate vaccine on drug-resistant Streptococcus pneumoniae. N Engl J Med 2006, 354:1455-1463.

5. Millar EV, O'Brien KL, Watt JP, et al.: Effect of community-wide conjugate pneumococcal vaccine use in infancy on nasopharyngeal carriage through 3 years of age: a cross-sectional study in a high-risk population. Clin Infect Dis 2006, 43:8-15.

6. Huijsdens XW, van Santen-Verheuvel MG, Spalburg E, et al.: Multiple cases of familial transmission of communityacquired methicillin-resistant Staphylococcus aureus. J Clin Microbiol 2006, 44:2994-2996.

7. Marcinak JF, Frank AL: Treatment of community-acquired methicillin-resistant Staphylococcus aureus in children. Curr Opin Infect Dis 2003, 16:265-269.
8. Fuller JD, Low DE: A review of Streptococcus pneumoniae infection treatment failures associated with fluoroquinolone resistance. Clin Infect Dis 2005, 41:118-121.

9. Clinical trial design for studies of otitis media. Presented at the U.S. Department of Health and Human Services Food and Drug Administration Center for Drug Evaluation and Research Anti-infective Drugs Advisory Committee, Gaithersburg, MD; July, 11, 2002.

10. Clinical trial design in acute bacterial sinusitis (ABS). Presented at U.S. Department of Health and Human Services Food and Drug Administration Center for Drug Evaluation and Research Anti-infective Drugs Advisory Committee, Gaithersburg, MD; October 29, 2003.

11. Gilbert K, Fine MJ: Assessing prognosis and predicting patient outcomes in community-acquired pneumonia. Sem Resp Infect 1994, 9:140-152.

12. Martinez JA, Horcajada JP, Almela M, et al.: Addition of a macrolide to a beta-lactam-based empirical antibiotic regimen is associated with lower in-hospital mortality for patients with bacteremic pneumococcal pneumonia. Clin Infect Dis 2003, 36:389-395.

13.• Song JH, Jung SI, Ki HK, et al.: Clinical outcomes of pneumococcal pneumonia caused by antibiotic-resistant strains in Asian countries: a study by the Asian Network for Surveillance of Resistant Pathogens. Clin Infect Dis 2004, 38:1570-1578.

Excellent example of the disconnect between in vitro and in vivo pneumococcal resistance with commonly used antimicrobials in a part of the world that has the worst incidence of drug-resistant pneumococci.

14. Pallares R, LiZares J, Vadillo M, et al.: Resistance to penicillin and cephalosporin and mortality from severe pneumococcal pneumonia in Barcelona, Spain. N Engl J Med 1995, 333:474-480.

15. Viladrich PF, Cabellos C, Pallares R, et al.: High doses of cefotaxime in treatment of adult meningitis due to Streptococcus pneumoniae with decreased susceptibilities to broad-spectrum cephalosporins. Antimicrob Agents Chemother 1996, 40:218-220.

16. Schaad UB: Predictive value of double tympanocentesis in acute otitis media. Pharmacotherapy 2005, 25:105S-108S.

17. Powers JH: Microbiologic surrogate end points in clinical trials of infectious diseases: example of acute otitis media trials. Pharmacotherapy 2005, 25:109S-123S.

18. Toltzis P: Comparison of amoxicillin with alternative agents for the treatment of acute otitis media in children. Pharmacotherapy 2005, 25:124S-129S.

19. American Academy of Pediatrics Subcommittee on Management of Acute Otitis Media: Diagnosis and management of acute otitis media. Pediatrics 2004, 113:1451-65.

20. Amsden GW: Interrelationship of pharmacokinetics/ pharmacodynamics: antibiotic dosing for the future. In Antibiotics and the Lung. Edited by Cazzola M, Blasi F, Ewig S. Leeds: Maney Publishing; 2004:1-12. 